\title{
CORRELATION OF MID-UPPER ARM CIRCUMFERENCE WITH BODY MASS INDEX AND WAIST-HIP RATIO IN HAEMODIALYSIS PATIENTS
}

\author{
Sujatha Banglore Bayer ${ }^{1}$, Faiza Banu Siddiqui², Shaikh Siraj Ahmed ${ }^{3}$ \\ ${ }^{1}$ Assistant Professor, Department of Anatomy, College of Medicine, Imam Abdulrahman Bin Faisal University, Dammam, Kingdom of \\ Saudi Arabia. \\ ${ }^{2}$ Assistant Professor, Department of Anatomy, College of Medicine, Imam Abdulrahman Bin Faisal University, Dammam, Kingdom of \\ Saudi Arabia. \\ ${ }^{3}$ Associate Professor, Department of Anatomy, College of Medicine, Imam Abdulrahman Bin Faisal University, Dammam, Kingdom of \\ Saudi Arabia.
}

\section{ABSTRACT}

\section{BACKGROUND}

Chronic malnutrition is one of the most significant problems in patients on Haemodialysis (HD). The body composition has a complex relation with clinical outcomes in these patients. Many anthropometric indices are available to measure the nutritional status and body composition. Mid-Upper Arm Circumference (MUAC) is a quick assessment anthropometric index of the nutritional status. We correlated MUAC with Height (HT), Weight (WT), Body Mass Index (BMI), Waist Circumference (WC), Hip Circumference (HC) and Waist-Hip ratio (WHR) in haemodialysis patients.

\section{MATERIALS AND METHODS}

Thirty patients undergoing maintenance haemodialysis for stage V chronic kidney disease were recruited. Age, gender, HT, WT, BMI, WC, HC, WHR, MUAC and body fat were recorded during the initial visit. Pearson correlation analysis was performed between MUAC and demographic and anthropometric indices.

\section{RESULTS}

The mean age of the patients was $48.03 \pm 11.81$ years. There were $21 / 30(70 \%)$ males and $9 / 30(30 \%)$ females. The duration of dialysis was $12 \pm 4$ months. MUAC significantly correlates with weight, body mass index, waist circumference, hip circumference, waist-hip ratio and body fat. It does not significantly correlate with either age or height.

\section{CONCLUSION}

MUAC correlates significantly both with abdominal adiposity (WC or WHR) along with overall adiposity (BMI).

\section{KEYWORDS}

Mid-Upper Arm Circumference, Abdominal Adiposity, Waist Circumference, Waist-Hip Ratio, Haemodialysis, Body Mass Index.

HOW TO CITE THIS ARTICLE: Bayer SB, Siddiqui FB, Ahmed SS. Correlation of mid-upper arm circumference with body mass index and waist-hip ratio in haemodialysis patients. J. Evolution Med. Dent. Sci. 2017;6(73):5183-5185, DOI: $10.14260 /$ Jemds/2017/1126

\section{BACKGROUND}

Protein energy malnutrition among dialysis patients and is associated with increased morbidity and mortality in patients on Haemodialysis (HD).(1) Causes of malnutrition in dialysis patients include low calorie or protein intake, stimulation of amino acid and protein degradation and stimulation of protein degradation. Early identification of malnourished patients and intervening at an early stage will improve the overall prognosis of the patients.(2) The simple methods of assessment of nutritional status include measurement of actual body weight, ideal body weight, Mid-Upper Arm Circumference (MUAC), Body Mass Index (BMI), subjective global assessment and bioimpedance.(3),(4) BMI is one of the best indicators of nutritional status in adults. (5) The BMI is a

Financial or Other, Competing Interest: None.

Submission 18-08-2017, Peer Review 31-08-2017,

Acceptance 02-09-2017, Published 11-09-2017.

Corresponding Author:

Dr. Sujatha Banglore Bayer,

Assistant Professor, Department of Anatomy,

College of Medicine,

Imam Abdulrahman Bin Faisal University,

Dammam, Kingdom of Saudi Arabia.

E-mail: sujathagmc@yahoo.com

DOI: $10.14260 /$ jemds $/ 2017 / 1126$ and diabetes. BMI is associated with stable haemodialysis and minimum mortality rates in patients less than 60 years. ${ }^{(6)} \mathrm{BMI}$ $\geq 30 \mathrm{~kg} / \mathrm{m} 2$ is associated with improved survival among chronic haemodialysis patients.(7) Low Body Mass Index (BMI) predicts poor survival in haemodialysis patients. ${ }^{(8)}$ However, BMI does not demarcate fat and muscle mass. ${ }^{(9)}$ Mid-Upper Arm Circumference (MUAC) is used to assess muscle mass, another anthropometric marker of adult nutritional status. There exists a significant correlation between MAC and the prevalence of malnutrition in patients.(10) MUAC fundamentally becomes a marker of malnutrition in patients for whom weight and height cannot be determined.

There exists a significant correlation between BMI and arm circumference.(11) The midarm circumference reflects the skeletal mass and the mid-arm muscle circumference (MAMC) measures the protein status in the body. A significant correlation exists between MAC and the prevalence of malnutrition in patients.(2) In the general population, waist circumference was noted to be a reliable predictor of visceral fat. Also, increased waist circumference was strongly associated with risk factors for cardiovascular disease.(12) Waist Circumference (WC), a nutritional marker, 
measures abdominal fat and is associated with mortality in haemodialysis patients.(7) We evaluated the correlation of mid-upper arm circumference with demographic and anthropometric indices in patients on maintenance dialysis at our hospital.

\section{MATERIALS AND METHODS}

This descriptive study was conducted at Gandhi Medical College Hospital, Hyderabad, during the years 2004-2006. Both male and female patients undergoing maintenance haemodialysis for stage $\mathrm{V}$ chronic kidney disease were recruited. Patients were excluded if they had any chronic liver disease, significant ischaemic heart disease, congestive cardiac failure, life-threatening arrhythmias, cardiomyopathy, coronary and cerebrovascular disease, chronic obstructive pulmonary disease and active autoimmune disease were excluded.

Demographic and clinical data were recorded from these patients. Height was measured using stature meter and weight using the calibrated weighing machine. BMI was calculated using formula weight (kg)/height (m2) (6). MUAC was measured using non-stretch tapes at the midpoint between the lateral acromion and distal olecranon process when arm flexed to 90 degrees at the elbow. WC was measured with a tape at midway between the lower rib margin and the iliac crest when the patient was standing in an upright position next to the stadiometer. HC was recorded at the maximum point of the buttocks. Body fat was noted by using bioelectrical impedance analysis.

\section{Statistical Analysis}

Data were entered in Microsoft excel spreadsheet 2003 statistical analysis was performed using GraphPad Prism (Version 4). The categorical data were expressed as proportions and percentages, and continuous variables were expressed as a mean and standard deviation. Pearson correlation analysis was performed between MUAC and demographic and anthropometric indices. A probability value of less than 0.05 was considered statistically significant.

\section{RESULTS}

A total of 30 patients undergoing haemodialysis were evaluated. The mean age of the patients was $48.03 \pm 11.81$ years; there were $21 / 30$ (70\%) males and $9 / 30$ (30\%) females. The duration of dialysis was $12 \pm 4$ months. There were $8 / 30(27 \%)$ were undernourished and remaining $22 / 30(73 \%)$ were normal body type as per international BMI standards. Data of the anthropometric indices in haemodialysis patients can be seen from Table 1. MUAC significantly correlates with weight, body mass index, waist circumference, hip circumference, waist-hip ratio and body fat. It does not significantly correlate with either age or height (Table 2).

\begin{tabular}{|c|c|c|c|}
\hline & Mean & SD & N \\
\hline Age & 48.03 & 11.81 & 30 \\
\hline Height & 162.00 & 9.79 & 30 \\
\hline Weight & 54.51 & 12.72 & 30 \\
\hline Body mass index & 20.75 & 4.07 & 30 \\
\hline MUAC & 23.08 & 3.80 & 30 \\
\hline Waist circumference & 81.07 & 12.28 & 30 \\
\hline
\end{tabular}

\begin{tabular}{|c|c|c|c|}
\hline Hip circumference & 86.83 & 9.97 & 30 \\
\hline Waist-hip ratio & 0.93 & 0.07 & 30 \\
\hline Body fat & 22.27 & 9.00 & 30 \\
\hline
\end{tabular}

Table 1. Descriptive Statistics of Demographic and Anthropometric Indices in Patients Undergoing Dialysis

\begin{tabular}{|c|c|c|c|c|}
\hline Pearson & $\mathbf{r}$ & $\mathbf{p}$ & Significance & $\mathbf{N}$ \\
\hline Age & -0.001 & 0.994 & No & 30 \\
\hline Sex & 0.165 & 0.385 & No & 30 \\
\hline Height & 0.3 & 0.107 & No & 30 \\
\hline Weight & $0.709^{* *}$ & $<0.0001$ & Yes & 30 \\
\hline Body mass index & $0.679^{* *}$ & $<0.0001$ & Yes & 30 \\
\hline Waist circumference & $0.777^{* *}$ & $<0.0001$ & Yes & 30 \\
\hline Hip circumference & $0.744^{* *}$ & $<0.0001$ & Yes & 30 \\
\hline Waist-hip ratio & $0.491^{* *}$ & 0.006 & Yes & 30 \\
\hline Body fat & $0.494^{* *}$ & 0.006 & Yes & 30 \\
\hline
\end{tabular}

Table 2. Correlation of MUAC with Demographic and Anthropometric Indices in Patients Undergoing Dialysis

\section{DISCUSSION}

Poor nutritional status among haemodialysis patients is a primary predictor of shorter survival time.(13) Nutritional assessment is a vital function of healthcare providers given preventing protein-energy malnutrition and provide adequate treatment to malnourished patients.(14) According to the National Kidney Foundation/Dialysis Outcome Quality Initiative Guidelines (National Kidney Foundation, 2002), the assessment of nutritional status in CKD patients on MHD should be made by integrating clinical, biochemical and anthropometric parameters.(15) In patients with maintenance haemodialysis, malnutrition and wasting are not only caused by inadequate nutrient intake, but also by catabolism due to inflammatory and noninflammatory conditions.

This study is meant to assess the nutritional status of haemodialysis patients. In our study, we found a negative correlation of MUAC with age suggesting a decrease in muscle mass with age in general population.(1) The reduction in muscle mass is termed as sarcopenia and it increases with age in haemodialysis patients.(3) Adult MUAC has long been known to reflect changes in adult body weight. We also observed a significant correlation of MUAC with weight. We also noted that MUAC is independent of the sex of the patient. A study reported that MUAC is associated with BMI, a simple measure of body size in hospitalised patients(16) and our study findings were similar to this report. BMI, a simple measure of body size, this measurement does not account for variation in body fat distribution and abdominal fat mass. ${ }^{(4)} \mathrm{A}$ study showed that BMI emerges as the leading predictive factor of malnutrition among our patients on dialysis followed by protein intake. Waist circumference, measures both intra-abdominal fat mass and total fat.(15) MUAC correlates significantly both with abdominal adiposity (measured by waist circumference or WHR) along with overall adiposity (as measured by BMI and body fat).(11) An earlier study has shown that waist circumference correlated well with the level of abdominal visceral adipose tissue than is WHR.(2) In our study, we also found that MUAC correlates so closely with BMI, WC, HC and WHR.

\section{Limitations}

This study was cross sectional and could not be generalised due to small sample size, the absence of serum albumin levels, fair assessment of fat mass and lean body mass. 


\section{CONCLUSION}

MUAC significantly correlates with weight, body mass index, waist circumference, hip circumference, waist-hip ratio and body fat.

\section{REFERENCES}

[1] Bonanni A, Mannucci I, Verzola D, et al. Protein-energy wasting and mortality in chronic kidney disease. Int J Environ Res Public Health 2011;8(5):1631-54.

[2] Koor BE, Nakhaie MR, Babaie S. Nutritional assessment and its correlation with anthropometric measurements in haemodialysis patients. Saudi J Kidney Dis Transpl 2015;26(4):697-701.

[3] Kondrup J, Allison SP, Elia M, et al. ESPEN guidelines for nutrition screening 2002. Clin Nutr 2003;22(4):415-21.

[4] Yamada K, Furuya R, Takita T, et al. Simplified nutritional screening tools for patients on maintenance haemodialysis. Am J Clin Nutr 2008;87(1):106-13.

[5] Flegal KM, Shepherd JA, Looker AC, et al. Comparisons of percentage body fat, body mass index, waist circumference, and waist-stature ratio in adults. Am J Clin Nutr 2009;89(2):500-8.

[6] Aoyagi T, Naka H, Miyaji K, et al. Body mass index for chronic haemodialysis patients: stable haemodialysis and mortality. Int J Urol 2001;8(8):S71-5.

[7] Salahudeen AK. Obesity and survival on dialysis. Am J Kidney Dis 2003;41(5):925-32.

[8] Leavey SF, Strawderman RL, Jones CA, et al. Simple nutritional indicators as independent predictors of mortality in haemodialysis patients. Am J Kidney Dis 1998;31(6):997-1006.
[9] Huang CX, Tighiouart H, Beddhu S, et al. Both low muscle mass and low fat are associated with higher all-cause mortality in haemodialysis patients. Kidney Int 2010;77(7):624-9.

[10] Brito BN, Llanos SJP, Ferrer FM, et al. Relationship between Mid-Upper arm circumference and body mass index in inpatients. PLoS One 2016;11(8):e0160480.

[11] Akpolat T, Kaya C, Utaş C, et al. Arm circumference: its importance for dialysis patients in the obesity era. Int Urol Nephrol 2013;45(4):1103-10.

[12] Sanches FM, Avesani CM, Kamimura MA, et al. Waist circumference and visceral fat in CKD: a crosssectional study. Am J Kidney Dis 2008;52(1):66-73.

[13] Owen WF, Lew NL, Liu Y, et al. The urea reduction ratio and serum albumin concentration as predictors of mortality in patients undergoing haemodialysis. $\mathrm{N}$ Engl J Med 1993;329(14):1001-6.

[14] Nunes TF, de Campos G, de Paula XSM, et al. Dialysis adequacy and nutritional status of haemodialysis patients. Hemodial Int 2008;12(1):45-51.

[15] National Kidney Foundation. K/DOQI clinical practice guidelines for chronic kidney disease: evaluation, classification, and stratification. Am J Kidney Dis 2002;39(Suppl 1):S1-266.

[16] Powell-Tuck J, Hennessy EM. A comparison of midupper arm circumference, body mass index and weight loss as indices of undernutrition in acutely hospitalized patients. Clin Nutr 2003;22(3):307-12. 